\title{
A Qualitative Inquiry into the Contextualized Parental Mediation Practices of Young Children's Digital Media Use at Home
}

Bieke Zaman, Marije Nouwen, Jeroen Vanattenhoven, Evelien de Ferrerre \& Jan Van Looy

To cite this article: Bieke Zaman, Marije Nouwen, Jeroen Vanattenhoven, Evelien de Ferrerre \& Jan Van Looy (2016) A Qualitative Inquiry into the Contextualized Parental Mediation Practices of Young Children's Digital Media Use at Home, Journal of Broadcasting \& Electronic Media, 60:1, 1-22, DOI: $10.1080 / 08838151.2015 .1127240$

To link to this article: http://dx.doi.org/10.1080/08838151.2015.1127240

Published online: 01 Mar 2016.

Submit your article to this journal $\pi$

Q View related articles 5

View Crossmark data $₫$ 


\title{
A Qualitative Inquiry into the Contextualized Parental Mediation Practices of Young Children's Digital Media Use at Home
}

\author{
Bieke Zaman $\odot$, Marije Nouwen, Jeroen Vanattenhoven, \\ Evelien de Ferrerre, and Jan Van Looy
}

\begin{abstract}
Technologies are increasingly adopted and used by young children at home. Parents play an important role in shaping their media use, keeping certain possibilities open for children to play, learn, and socialize while limiting others. Nevertheless, the literature on parental mediation of young children's media use is scant. In this article, we describe a qualitative, mixed-method study involving 24 parents and 36 children aged 3 to 9, and focus on the contextual factors that shape (transitions between) parental mediation practices. The results point to the emergence of new manifestations of parental mediation and provide evidence of their dynamic, often paradoxical nature. In particular, the insights on distant mediation, various buddy styles, and participatory learning, as well as the value of a wholeness approach for understanding children's conditions for media engagement, suggest new prospects for parental mediation literature.
\end{abstract}

The mediation of children's media use has radically changed since households have started to appropriate digital technologies in everyday life. Parents are increasingly dealing with digital media they did not grow up with, and

Bieke Zaman (Ph.D., KU Leuven, Belgium) is an assistant professor at iMinds-CUO/IMS, KU Leuven, Belgium. Her research focuses on the uses, experiences, and practices of digital media in families with young children.

Marije Nouwen (M.A., KU Leuven, Belgium) is a researcher at the Centre for User Experience Research, KU Leuven, iMinds, Belgium. Her research interests include children and digital media, and ethnography in $\mathrm{HCl}$.

Jeroen Vanattenhoven (M.A., Group T, Belgium) is a senior researcher at the Centre for User Experience Research, KU Leuven, iMinds, Belgium. His research activities focus on Smart TV and Second Screen experiences.

Evelien de Ferrerre (M.A., KU Leuven, Belgium) is a doctoral candidate at the Leuven School for Mass Communication Research, Belgium. Her research interests include media psychology and sociology, in particular mediation in families and mediatization in organizations.

Jan Van Looy (Ph.D., KU Leuven, Belgium) is an assistant professor at iMinds-MICT-Ghent University, Belgium. His research focuses on the use and experience of digital games and immersive media.

Color versions of one or more of the figures in the article can be found online at www.tandfonline.com/hbem. 
challenged to seek a balance between minimizing children's exposure to risks while equally facilitating digital opportunities. Notwithstanding the increased adoption and use of technologies by young children (Chaudron, 2015; Plowman \& McPake, 2013), the available literature on parental mediation (PM) of young children's digital media use is relatively scarce. Moreover, there is a dearth of research that gives full account of the contextual factors that determine PM practices in the family home.

Therefore, this article contributes to the study of PM with a qualitative inquiry into the contextual factors that shape PM practices of young children's digital media use. Based on the findings from this exploratory, mixed-method study involving 24 Belgian families with children between 3 and 9 years old, we suggest new manifestations of PM strategies. Also, we emphasize their dynamic, and often paradoxical nature.

By providing in-depth insights into the complexities of PM practices, we complement the findings from previous work, which are mainly quantitative in nature (Clark, 2011a, 2011b; Plowman, 2014; Ólafsson, Livingstone, \& Haddon, 2014). Although important precursors of PM have already been extensively reported on (see, e.g., Connell, Lauricella, \& Wartella, 2015; Gentile, Nathanson, Rasmussen, Reimer, \& Walsh, 2012; Mendoza, 2009), it is still unclear how and why these precursors result in particular PM manifestations. Accordingly, the importance of accounting for the influence of the home context in shaping children's experiences with technologies is increasingly put forward (Nathanson, 2015; Plowman \& McPake, 2013).

Furthermore, previous work on PM has primarily focused on television use (Mendoza, 2009; Valkenburg, Krcmar, Peeters, \& Marseille, 1999) or on children aged 12 and older (Holloway, Green, \& Livingstone, 2013; Nikken \& Jansz, 2014; Ólafsson et al., 2014). Although some recent studies have started to account for the mediation of children's media use beyond television, such as video gaming (Nikken \& Jansz, 2006, 2014; Nikken, Jansz, \& Schouwstra, 2007; Shin \& Huh, 2011) or Internet usage (Eastin et al., 2006; Lee, 2013; Livingstone \& Helsper, 2008), the focus has remained limited to one particular digital medium (Livingstone \& Helsper, 2008; Shin \& Huh, 2011). Nonetheless, as an array of devices facilitates similar digital activities (Jenkins, 2004), it is increasingly meaningful to study PM with regard to various media devices and platforms, as well as account for the synergies of media usage patterns (Rodino-Colocino, 2007). Moreover, given the increased diversity of digital devices at home and their interactive, immediate, social, and ubiquitous characteristics, media scholars should investigate if, how, and why PM practices evolve along with the newest digital possibilities.

\section{Parental Mediation Literature}

For more than three decades, there has been research on PM of children's television use (Warren, 2001). These studies have emerged from the tradition of 
media effects research (Clark, 2011b), concerned with "any strategy parents use to control, supervise, or interpret content" (Warren, 2001, p. 212) to mitigate the negative effects on children's physical, psychological, and emotional health (Mendoza, 2009). Overall, previous research has repeatedly put forward three strategies for the mediation of children's television viewing, and more recently also regarding Internet use and video gaming. These strategies are restrictive mediation, active mediation, and co-use. Studies from the media literature have mainly investigated PM by focusing on one particular medium, whereas the parenting literature has approached this topic mainly with a general take on media (Warren, 2001). The parenting literature, too, has pointed to restrictive and active mediation strategies, but categorized these as two manifestations of proactive monitoring, termed cocooning and prearming, respectively. Co-use strategies were not referred to, but instead, the mediation strategy of deference has been introduced as a third manifestation of proactive monitoring (PadillaWalker, Coyne, Fraser, Dyer, \& Yorgason, 2012). Most recently, two new categories have been introduced in the pivotal article of Nikken and Jansz (2014), the first study that has dealt with PM of digital media use in families with young children. In what follows, we further describe the similarities and differences in PM for various media and with respect to young children, focusing on the age spectrum of 3 to 9, versus older children, mainly hitting upon ages of 13 and older. We will, however, limit ourselves to studies that have included Western populations and advanced countries in order to sketch a comparable interpretative framework for our study.

First, restrictive mediation has been characterized by the rules enforced by parents to limit and control children's media usage. The categories of restrictions are similar for children along the age spectrum, stipulating the amount of time ("how long?"), content ("what?"), and moment ("when?") (Gentile et al., 2012; Nikken \& Jansz, 2014; Warren, 2001). In research on elementary school children's television viewing, restrictive mediation also manifested as a way to reward or punish (Warren, 2001). Research on the mediation of video gaming of children aged 8 to 18 has shown that restrictive mediation is mainly applied to avoid negative effects (Nikken \& Jansz, 2006). In general, restrictive mediation is likely to be applied towards older children's Internet use (Nikken \& Jansz, 2014) and among parents who are concerned about the negative effects of video games (Nikken \& Jansz, 2006).

Second, active mediation refers to instructive or evaluative conversations in order to explain, discuss, and/or share critical comments-for instance, by purposefully explaining media content in words children can understand (Gentile et al., 2012; Warren, 2001). The instructive approach aims at an educational outcome, such as teaching children technical aspects, whereas the evaluative approach aims at a normative outcome, such as expressing (dis)approval. In general, active mediation is most likely to occur in families with older children, primarily to protect them against the risks of social networking sites (Sonck, Nikken, \& de Haan, 2012) and video gaming (Nikken \& Jansz, 2006). In families with young children, it is more 


\section{Journal of Broadcasting \& Electronic Media/March 2016}

likely to occur among parents with positive beliefs towards their children's Internet use than among parents with negative beliefs (Nikken \& Jansz, 2014).

Finally, co-use as a mediation style has often been termed "co-viewing" in research on the mediation of television viewing or "co-playing" for video gaming. It entails shared media activities, often driven by a common interest (Nikken \& Jansz, 2006; Valkenburg et al., 1999) and sometimes resulting in content discussions (Gentile et al., 2012). Mendoza (2009) has shown that parents who apply co-use without discussion for television viewing are likely to have a positive attitude towards the content. Nikken \& Jansz' (2014) results have also shown that positive beliefs about the effects of young children's Internet use are likely to go hand-in-hand with co-use mediation styles.

Most recently, Nikken and Jansz (2014) introduced two additional strategies for the mediation of Internet use in families with young children aged 2 to 12, namely technical safety guidance and supervision. Technical safety guidance incorporates technology-supported safety measures such as anti-virus programs or spam filters, as well as applications that are purposefully designed to protect children's safety, like black-/whitelist filters. This strategy is mostly exerted by computer literate parents.

The second additional strategy is supervision "whereby the child is allowed to go online alone but with the reassurance that he or she is under direct supervision of a nearby parent" (Nikken \& Jansz, 2014, p. 259). It is often combined with housekeeping activities, and the child is only interrupted or assisted when necessary. Supervision is more common in families with children younger than 12, and especially in families with fewer computers, when the computer is used in the living room and negative effects of children's Internet usage are expected (Nikken \& Jansz, 2014). To some extent, supervision shares characteristics with the deference strategy as reported in the parenting literature. Both imply monitoring behavior where parents do not intervene as long as they don't observe negative effects. However, deference has only been described for parents with adolescents who are granted autonomy and trust without these parents being overindulgent (Padilla-Walker et al., 2012).

In the current study, we focus on parental efforts toward diverse uses of digital media by young children and adhere to a broad categorization of mediation, including strategies used to mediate and prevent negative media effects. We equally foreground strategies parents employ to incite positive experiences, and are responsive to Clark's (2011b) call to empirically investigate the emergence of a potential new category, that is participatory learning. Our literature review has nonetheless shown that insights on mediation practices in families with young children are far from complete and would benefit from in-depth analyses revealing their contextual factors. Hence, our research questions (RQs) are:

$\mathbf{R Q}_{\mathbf{1}}$ : Which strategies do parents apply in the home context to mediate young children's digital media use and what are the characteristics?

$\mathbf{R Q}_{2}$ : What are the contextual factors influencing parental mediation practices? 


\section{Method}

\section{Participants}

A total of 24 Flemish families living in Belgium and with children between 3 and 9 years old participated in the study. Families were recruited based on variability in residency, income, and family composition. The incentive for each participating family was a voucher worth 50 Euros. A total of 22 mothers and 2 fathers $\left(M_{\text {age }}=\right.$ $36, S D_{\text {age }}=5.16$ ) agreed to participate allowing us to include data on 49 children of which 36 were between 3 and 9 years old. Details on the participating families were gathered via a recruitment questionnaire and diary; for an overview we refer to Appendix 1. In the results section, we will make reference to a particular family with an abbreviation that comprises information on child(ren)'s gender and age(s), respectively. To illustrate, the reference for the first family listed in Appendix 1 with two children aged 7 and 10, is Family_A (girl7, girl10).

\section{Procedure}

Data collection, including a pilot test, took place from May to July 2013. Participating families were visited twice. The first visit began with a description of the study's goals, reassurance about confidentiality, and the signing of the informed consent forms. Participants were trained in using the diary by means of a discussion of two pages containing relevant example activities and instructed to fill out the blank diary pages for 10 days. Figure 1 shows the introductory page with space to provide information on the family composition as well as the number and type of devices owned. The main diary template spanned two pages (see Figure 2), requesting information on time and duration (start and end), location, title/name, type of media-related activity, device, person(s) involved (including the role of the parent), and additional remarks. Finally, if applicable, parents could write down general notes on the diary's last page.

The second visit consisted of an interview with the parent, as well as a simulation wherein we invited the child(ren) to demonstrate the use of a digital medium of choice and the parent(s) to mediate their child(ren)'s media usage and end the activity. Both were instructed to act as they would normally. In probing for more explanations during the interviews, we were not only led by the a priori defined interview guide but also by the events from the diary and simulation. The second home visit lasted between 45 and 75 minutes.

\section{Data Analysis}

The interviews and verbal accounts of the simulation were audio recorded, transcribed, and proofread before the directed content analysis in NVivo 10. Data from the interviews, diaries, and simulations were triangulated in order to account for 
6 Journal of Broadcasting \& Electronic Media/March 2016

Figure 1

Example Diary Introductory Page.

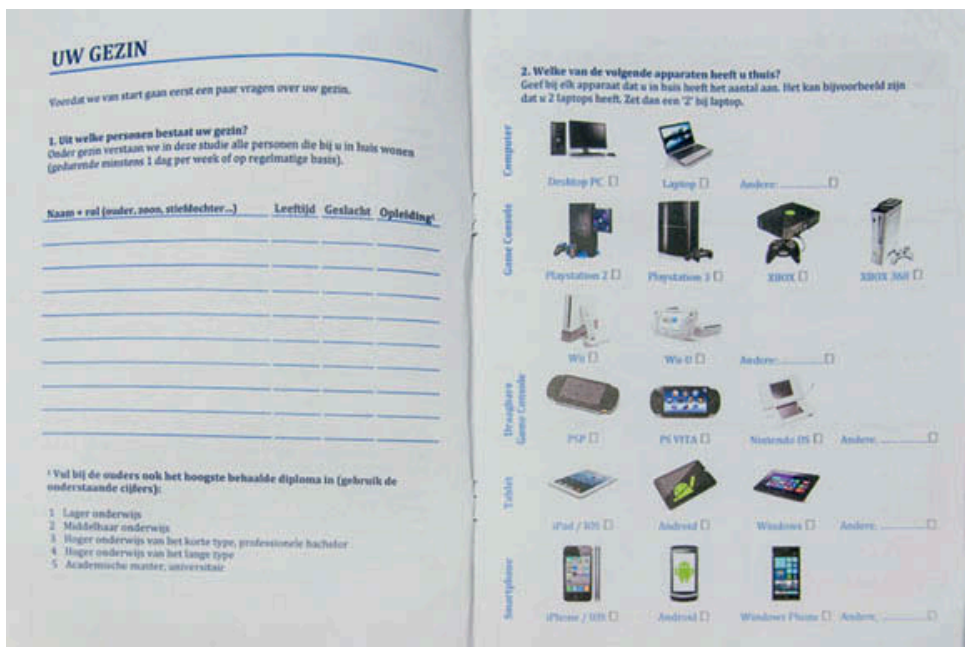

Figure 2

Example Diary: Main Template for Daily Entries.

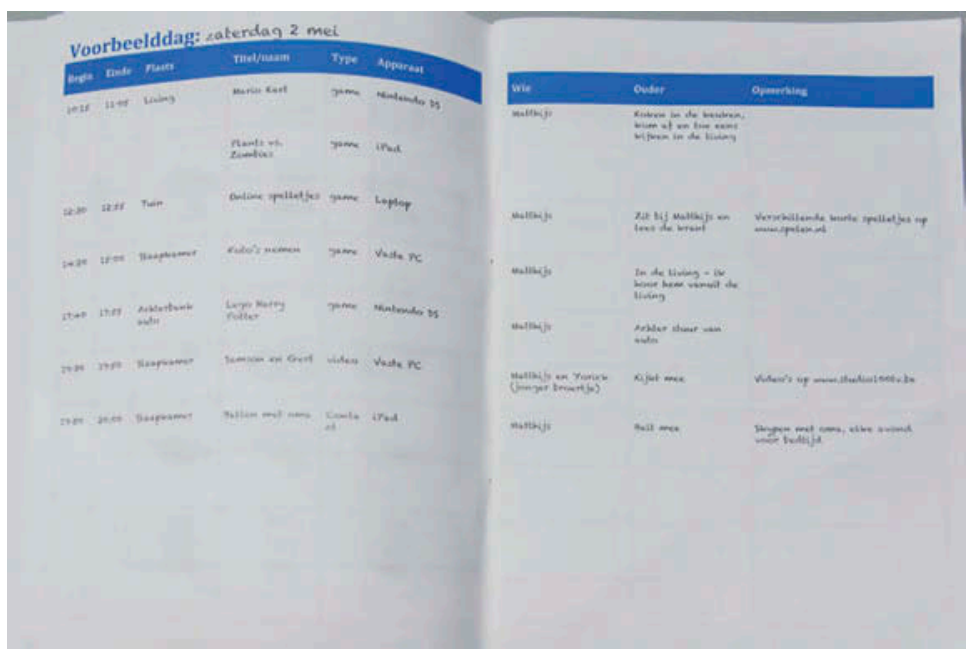


Figure 3

\section{Concept Map Displaying Parental Mediation Strategies and Contextual Factors.}

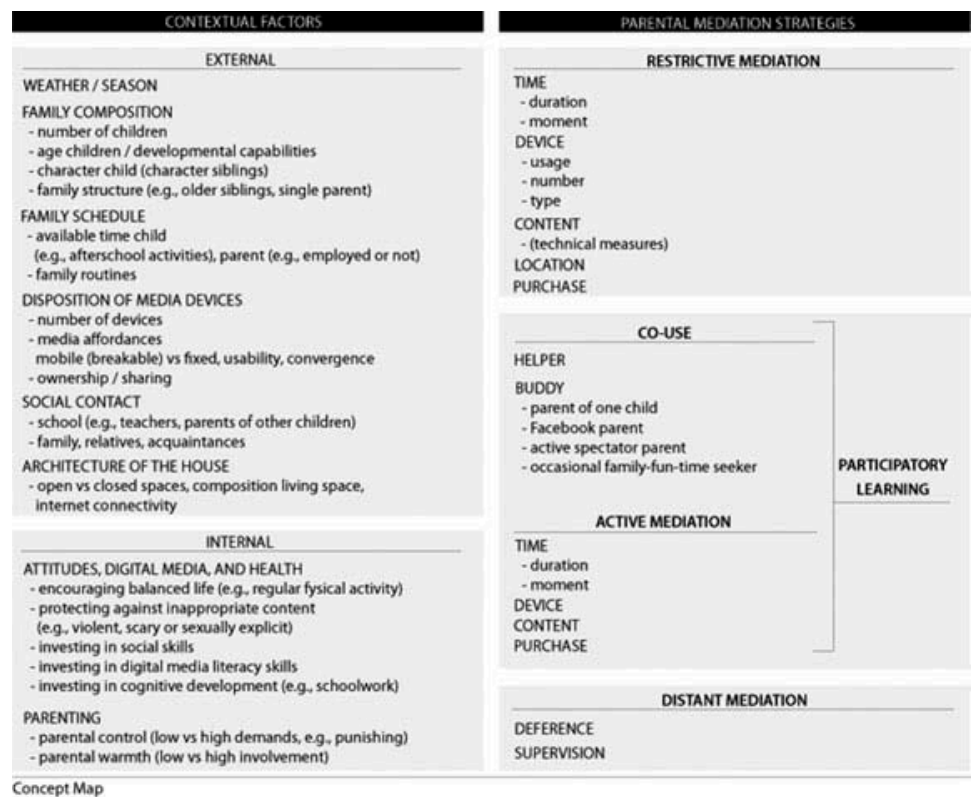

possible social desirability effects and construct a valid coding scheme. In the first round of open coding, we focused on sections that could characterize patterns of mediation strategies as found in the previous literature, sections that could suggest new strategies, and sections hinting at contextual factors. Then, during two iterations of axial coding, we reviewed the data and compared the mediation practices and contextual factors in order to discover similarities and differences within codes as well as their (hierarchical) relations. This was an iterative process where we first specified codes individually, followed by discussions among the authors to agree upon a concept map (Figure 3).

\section{Results}

The concept map in Figure 3 summarizes the results of our study. It presents mediation strategies that were referred to in the previous literature as well as new strategies that emerged from our data $\left(R_{1}\right)$. Additionally, it shows both external and internal contextual factors that elicit (transitions between) mediation practices $\left(\mathrm{RQ}_{2}\right)$. 


\section{Journal of Broadcasting \& Electronic Media/March 2016}

\section{Restrictive Mediation}

In total, the results revealed five types of restrictive mediation relating to time, device, content, location, and purchase.

Time Restrictions. The results pointed to restrictive time measures that were directed at the moment of media usage (e.g., limiting digital play in favor of outdoor play when the weather is nice) and the duration (e.g., imposed via kitchen timer). Duration restrictions were especially pertinent to children in primary school, as illustrated by the mother in Family_K (girl7, boy8, boy11): "normally they play about half an hour, [...] maximum one hour," and less applicable to toddlers because their limited attention span already instilled activity variation without parental intervention. Parents ended their children's media use either via a communicative approach, by allowing their children to have a say (i.e., transition to active mediation, see further) or via a non-communicative approach where children were expected to obey immediately. In the latter, parents often took strong measures, such as turning off the device, if children acted adversely.

The participating parents motivated their restrictions by assessing the sum of activities children engage in, including non-digital activities. They made decisions in favor of children's health, underscoring the importance of physical activities and, more generally, a healthy and varied program of leisure activities. Some parents restricted gaming but allowed television use before bedtime, hereby preferring a medium that would keep the child relaxed. Furthermore, educational concerns seemed to outweigh entertainment in the mediation of children of school age, with parents restricting digital media use until homework was finished, restricting technology use on weekdays, or only allowing educational media content during weekdays. The latter dynamics illustrate that time, content, and device decisions are often mutually dependent and influenced by the particularities of both parents (e.g., beliefs) and child (e.g., primary school versus preschool). Other contextual factors were the family schedule (e.g., bedtime routines), disposition of media devices (with particular affordances), and family composition. For instance, the diary and interview of Family_A (girl7, girl10) showed that this mother regularly allowed her youngest child to watch television so she could assist in the oldest child's homework.

Device Restrictions. Device restrictions were mostly set up to control usage and avoid children changing "something in the settings by accident" (mother, Family_E girl0, boy6), breaking the devices, or as a punishment. It often implied that children were not allowed to play by themselves or walk around with portable devices, that devices were kept away from children, or that they had to seek permission to use them.

Other restrictions concerned the number and type of device(s). For instance, parents favored the use of alternative devices when it was strongly personally appropriated (e.g., parent's mobile phone), when used for work (e.g., laptop), or when it concerned a 
device that an older sibling had bought him-/herself. Mediation differed according to parental judgment on the appropriateness of the devices in terms of usability and children's developmental capabilities. To illustrate, "The tablet is a handy device. They take it with them. The laptop, too, but still, the tablet is more compact and easier for them" (mother, Family_D boy4, girl6). Also, "the tablet is more convenient; you can't do much wrong. Everything has been set up, but the computer... you can always click on something" (mother, Family_R boy3, girl4, girl7).

Content Restrictions. Many parents expressed concerns regarding inappropriate (i.e., violent, sad, scary, or sexually explicit) content. The mother of Family_H (boy7) put it simply: "He is only 7 . You can't expect them to be 18 already." Parents were likely to discuss confrontations with undesirable content, delineating a transition to active mediation (see further). The mother of Family_R (boy3, girl4, girl7) had installed technical restrictions on the family's laptop as a preventive measure. However, she and her husband had quickly decided to uninstall these because they hampered their own online activities. The interviews further showed that the majority of parents asserted not (yet) having installed technical safety measures but taking this into consideration when their children grow older, start using digital media more intensively, and/or when they are more likely to consume violent content, "like games where they shoot each other" (mother, Family_A girl7, girl10) or "less innocent" content (mother, Family_F girl3, boy6), like "chatting" (mother, Family_E girl0, boy6).

Location Restrictions. All participating parents imposed location restrictions as a measure to safeguard children's health, protect the device from damage, or facilitate the distant (auditory/visual) monitoring of children's media activities. To illustrate, the mothers of Family_M (boy0, girl3) and Family_R (boy3, girl4, girl7) expressed their concerns about the negative effects of using mobile media on the couch, referring to neck pain and fertility problems, respectively. The latter mother added a pragmatic issue: "On the couch it is so comfortable. If I then ask to stop, it will become more difficult for them [...], whereas at the table they are more easily tired of it by themselves." Other contextual factors that shaped (exceptions on) location restrictions were the strength of Internet connectivity, family schedule, and the composition of living space.

Purchase Restrictions. Several parents stated they had set rules in place to safeguard their budget, such as allowing "free apps only" (mother Family_D boy4, girl6), or stipulated how to save up to buy new devices or content. For instance, the mother in Family_R boy3, girl4, girl7 explained why they had bought a tablet: "The tablet of my mother [the child's grandmother] had many applications whereas with our [Nintendo] DS we always had to buy games, the same goes for the [Nintendo] Wii. And those [tablet] apps, we already found them super." This rule-setting often switched to active mediation when parents allowed input from the child and rule negotiation (see further). 


\section{Co-use}

Two manifestations of co-use emerged from the data, namely the parent as helper and buddy.

Helper. Parents who acted as a helper guided children when they used a digital medium for the first time or when they encountered problems, for instance when children accidentally closed a Web browser or could not achieve a satisfying game result. "They usually don't play alone. [...] They're not familiar with it, so we do it together. [...] We help them out for a short while" (mother, Family_D boy4, girl6). Many parents asserted that their involvement decreased when their child became more experienced, linking mediation practices with children's (digital literacy) development.

Buddy. The parent as buddy enjoyed sharing certain media activities for recreational purposes. Our results revealed four manifestations of buddies, namely the parent of one child, the Facebook parent, the occasional family-fun-time seeker, and the active spectator.

Parent of One Child. Parents with only one child were often profoundly involved in their child's digital activities, which usually evolved into practices of active mediation or participatory learning (see further). Supposedly, these parents took up the role of the "missing" sibling. They enjoyed shaping digital experiences and collaborative decision-making. For instance, in Family_O the mother seemed to shape her 9-year-old son's game experiences considerably:

He comes and shows me: "I just constructed this [with Minecraft], what do you think?" Then I get to choose whether it should be in glass or with which stones. Or he asks me what kind of house I'd like.

Facebook Parent. Media consumption patterns of adults, and in particular Facebook use, seemed to shape children's digital activities. For instance, in Family_P (boy5, girl5, boy8), children regularly played online games like Bejeweled or Jetpack Joyride via the mother's Facebook account. In the diary, she explained this: "As my laptop is also being used professionally [...] the rule is, only games via mummy's Facebook account." Equally, in Family_S (boy8), the father explains that his son "is allowed to co-view his [i.e., the father's] Facebook feeds," and this "especially when there are soccer-related updates." Parents clarified their children had started showing interest because of their own Facebook activity. "He [i.e., 6-year-old son] saw me playing games the other day and wanted to try it out himself, too. Now he plays Panda Jam, [...], Farm Saga, a kind of Candy Crush, and Angry Birds, too" (mother, Family_F girl3, boy6). In some families, Facebook co-use became part of household routines. 
What we sometimes do is Bejeweled [... I I actually play that a lot. And he [son, age 8] also plays this game now and then. Every day you have to do a jackpot to gain extra points and then everybody starts guessing how many symbols we will have. It's a tradition to do every evening. (mother, Family_P boy5, girl5, boy8)

Occasional Family-Fun-Time Seeker. This manifestation of a buddy refers to parents who enjoy sharing digital media experiences with their children occasionally. For instance, in Family_B (girl0, girl8), the oldest daughter sometimes asks to go on YouTube, to which the mother reasons "when there is time in the evening, we sometimes do it together. It's fun." This manifestation of co-use seems to be related to parents' intentional investment in family bonding, as illustrated in the following interview excerpt:

If they do something together with the parents, it is not like "go away and entertain yourself." No, I am convinced that these are moments that you have to share with your child and with your family. Sending your children away just to get rid of them, that's not the rule here. (mother, Family_D boy4, girl6)

Active Spectator Parent. This manifestation occurred whenever children initiated interaction to show or share achievements and experiences. Parents then became spectators, driven by a genuine interest in and motivation to enjoy joint media activities. These intense but relatively short moments of involvement seemed to compensate for moments when parents had to multitask e.g., when working late, dividing attention among siblings, or, as illustrated in Family_O (boy9)'s diary, when switching regularly between "household activities," "allowing the child to demonstrate something," and "playing together."

\section{Active Mediation}

Our results showed that active mediation related to parent-child discussions with regard to time, device, content, and purchase.

Time. Time-related active mediation deals with the moment or duration of media usage. Parents often engaged in discussions to negotiate or justify their, typically restrictive, mediation practices. They link this to family practices, e.g., "because dinner is ready" (mother, Family_C boy6, boy8), or they believe that children should engage in a healthy and varied program of leisure activities, as illustrated by the mother in Family_G (girl3) "when she wants to watch television after school, I say no if the weather is nice. We are going to play outside, and I do explain this to her."

Device. Our results showed that parents regularly had to compensate for children's lack of media experiences or developmental issues. Therefore, they had 
to engage in instructive discussions or demonstrations to teach (the risks involving) technical issues, like download or installation procedures. Often, these mediation practices made parents switch to instances of co-use, for example when explaining how to proceed in a game (mother, Family_K girl7, boy8, boy11) or demonstrating how to use the mouse (mother, Family_M boy0, girl3). Alternatively, it could also end in instances of participatory learning when parents and children figured out technical issues together (see further).

Content. Discussions related to media content primarily occurred to avoid or mediate harm from inappropriate content. For example, in Family_I (boy8), the mother explained, "And then I ask him, "explain it to me" [...]. If there is violence in it [i.e., game], then I discuss this with him, explaining I do not like it." Content discussions were often elicited after children had learned new media opportunities from school, relatives, or friends, or after instances of being an active spectator. Many parents took the child's perspective into account and adjusted their approach because they didn't "want to point certain things out to her that she probably didn't see" (mother, Family_B girl0, girl8), trusted their children, relied on their interpretation, or because they wanted to observe the effects on their children first. Active mediation did not necessarily result in restrictions, even when parents disapproved of the content, as illustrated by the simulation of the mother and son in Family_O (boy9) that resides on trust in the child's judgment.

I don't know what you have to do here. I will have a look right now. Ah, with catapults [...], bats [...]. Yeah, I don't know. Explain to me the purpose of this game. Ah, you are stoning them? I don't really find this okay. But yeah [...] I let him play for a little while. It looks okay, kind of. [...] He knows that I don't like that. [...] He also tells me: "It's only a game, Mom."

Purchase. The data revealed instances of media purchase discussions. Many parents asserted justifying or negotiating purchase restrictions (see earlier), such as questioning the investment in a gaming console when they already had a tablet with access to a variety of free or low cost games. Alternatively, parents associated it with parental rewards, like in Family」 (boy4, boy7, boy10): "and they had a play date and found these [game consoles] interesting. Then I said, you know what, you get it for your birthday."

\section{Participatory Learning}

Our results provided empirical evidence of the strategy of participatory learning. It involved characteristics of co-use and active mediation and manifested itself mainly among parents who wanted to invest in their children's and/or their own knowledge and skills. On the one hand, it was directed at operational learning and considered as an investment in acquiring digital literacy skills for child and 
parent, as illustrated by the following interview quotes "I find it important that she develops the related skills" (father, Family_W girl3) and "It is just some kind of a phenomenon, a digital era. [...] You can't stop it. [...] I keep up with the times" (mother, Family_C boy6, boy8). This type of learning is further exemplified in Family_L (boy8)'s simulation in which mother and child collaboratively figured out how to set up a digital medium:

Child: Do I need this CD?

Parent: Good question. I will have a look; don't know whether it will work. Will try to find it. Here, do you see? Application not found. I have to install it first.

Child: Here? It worked for my teacher. [...]

Parent: Can you just look for the games and play instead of using the disc?

Child: Fine, I eject the CD first, right mum?

On the other hand, families also collaboratively learned through media, hereby extending the understanding of the world (i.e., not limited to media-related knowledge per se), such as instructing "to Google this, and then you know what it means" (mother, Family_L boy8).

\section{Distant Mediation}

The results pointed to instances wherein parents kept aloof from children's media usage while still keeping an eye on what is happening. We termed this distant mediation, as an umbrella term for deference and supervision.

Deference. Deference occurs when parents make the deliberate choice not to intervene and grant trust and autonomy to their children, expecting them to act responsibly. This was evidenced in our data as parents often said to keep informed from a distance (e.g., regularly checking the screen from nearby location) and maintain a considerable level of involvement while granting autonomy to the child. "When I am in the kitchen, I quickly go back and forth. But usually, he [son, aged 8] looks up the same things. So I am fairly at ease" (mother, Family_P boy5, girl5, boy8).

Supervision. Supervision is associated with parental efforts to allow children to use digital media independently but under direct parental supervision. Typically, it is a necessity because parents have to multitask with housekeeping activities. Our data pointed to several instances of supervision and the relation with external factors such as the limited available time of parents and welcoming digital media as a "babysitter." The simulation in Family_D boy4, girl6 illustrated transitions between co-use and supervision. We observed both parents actively engaging in their children's digital game experiences, but once their children became less in need of help, they continued their household-related practices. They did, however, 
deliberately remain within hearing distance to be able to easily switch back to their role of helper whenever necessary.

\section{Discussion and Conclusion}

The current study contributes to the body of research on PM with in-depth insights into the contextual factors that shape various mediation practices in families with young children. Additionally, it points to the emergence of new manifestations and transitions between them.

Concerning restrictive mediation, this study identified, in line with previous research, content and time restrictions exerted in order to avoid negative effects. Additionally, our results identified three new manifestations related to device, location, and purchase restrictions, hereby revealing other determining factors such as the fear of broken devices or the need to find ways to punish the child. Furthermore, in our data, technical measures were associated with content and device restrictions. Further work is needed to (re)consider the category of technical safety guidance, questioning whether it merits a separate mediation style. We argue that as long as the majority of technical measures afford time, content, or device restrictions, it is not surprising to see a common ground with restrictive mediation practices.

In addition to confirming the existence of co-use and active mediation strategies, this study also suggested interrelationships and nuances that have remained underdocumented in previous studies. The topics of discussion in active mediation are only partly in line with previous work on PM of television viewing (wherein content discussions predominated) and mediation of older children's digital media use (that has reported on content and device-technical discussions). Our results revealed that parents also discussed time and budget decisions to justify or negotiate the rules, hereby often pointing to transitions between active and restrictive mediation.

Our notion of co-use is in line with previous work (e.g., Nikken \& Jansz, 2006; 2014; Valkenburg et al., 1999) in that we also found that positive motivations like seeking shared media enjoyment can induce co-use. However, this study also pointed to new practices, making a distinction between the parent as helper and buddy. We believe that by having accounted for a variety of digital media activities and contextual factors, we were able to reveal various particular buddy manifestations and complement the insights from previous PM studies. Our findings indicated that buddy practices could result from intentional actions (e.g., high parental involvement, family quality time) as well as routine and family practices (e.g., parent's Facebook use or older siblings' media preferences inciting new media opportunities or risks).

Furthermore, our results suggested that co-use and active mediation were strongly interwoven. We even found empirical evidence that several of their characteristics merged when parents learned from or together with their children. We made use of Clark's (2011b) term, participatory learning, and delineated this as a fourth mediation strategy. Whereas to some extent, the instructive role of the parent has been put 
forward before (see e.g., Plowman \& McPake, 2013), the involvement of children in our study proved relevant in the refinement of PM theory as it revealed that children, too, initiated instructive discussions. Because of the importance of media literacy skills to securely participate in today's digital world, we strongly encourage future researchers to pay more attention to this mediation practice. In equal merit of further research attention is a better understanding of these manifestations in the light of the sociology of childhood (Clark, 2011b), taking into account children's agency both in relation to and beyond media use. We hereby reopen the debate on the appropriateness of the term "mediation" as parents do more than simply mediating negative media effects (Clark, 2011b; Padilla-Walker et al., 2012).

We identified a fifth mediation style, distant mediation, which aggregates two previously defined monitoring practices, namely deference and supervision. From the parenting literature, we learned that some parents monitor adolescents' behavior while deliberately granting them trust and autonomy, referred to as deference (Padilla-Walker et al., 2012). Supervision was used in the media literature and typically described as a necessity when parents have to multitask (Nikken \& Jansz, 2014). We have chosen to use a new umbrella term, distant mediation, because our data pointed to varied nuances of monitoring young children's media use and suggested the existence of a less hierarchical role for the parent, who is no longer expected to always take the lead, as Clark (2011b) has hypothesized.

Overall, this study revealed the dynamic and often paradoxical nature of PM. Clearly, parents' mediation practices depend on contextual demands, which evolve over time (see e.g., the rapidly changing popularity of a particular content or device) and vary between locations (e.g., more relaxing rules in the car or restaurant). Under certain circumstances, technologies serve as a substitute (e.g., as a "babysitter"), while under other they form the subject of parent-child interactions. PM practices also unfold differently depending on whether it precedes, happens during or after media use, and whether the parent or child is the initiator. In terms of motivations, we learned that although parents don't like their child to be too absorbed in digital play at the expense of a healthy balance in leisure activities (e.g., safeguarding sufficient time for physical activities and outdoor play), exceptions are allowed when media usage serves educational goals or is a convenient way of occupying the child. Parents believe that they cannot counter the advent of digital media and should, instead, keep up with the changing technology landscape. Even when holding negative attitudes towards digital media penetrating the home environment, parents seem to acknowledge beneficial uses. Surprisingly, our findings showed that parents express concerns regarding inappropriate content, but at the same time believe their young child does not have bad intentions when engaging with digital media and therefore is less likely to encounter risks. In general, they trust their young child(ren), consider their media preferences and uses as innocent, and believe parental mediation efforts suffice to remediate potential harmful effects. Parents do, however, anticipate more concerns as children grow older, become more digital media literate, and independent. 
Given the dynamic nature and situatedness of PM practices, we argue that further theoretical refinement of PM theory benefits from a cultural-historical "wholeness approach" that "includes a global-local dialectic in which childhood and children are seen in interdependent relation to their activities, institutional practices, and societal conditions" (Fleer, Hedegaard, \& Tudge, 2012, p. 9). In this study, we focused on the localized practices within the family. More particularly, we described how the conditions created for children are shaped by and are shaping family members' engagement in the (media) world, and hinted upon influences from outside the home context, like those from school, hobbies, relatives, and friends. The importance of accounting for contextual and social practices in the investigation of how people appropriate technologies and ascribe meaning to them, is in line with domestication theory (Silverstone \& Hirsch, 1992). Instances of two-way interactions between parent and child merit special attention in future work. Our results on participatory learning and the various buddy styles suggest the existence of less hierarchical and less riskavoidance, more opportunity-seeking mediation styles (see also e.g., Clark, 2011 b) or even reversed socialization relationships through processes of bottom-up technology transmissions (Correa, 2014). Moreover, this study exemplifies how the precursors of PM that have been extensively reported, like the demographic variables of parent and child (see e.g., Gentile et al., 2012; Mendoza, 2009; Nikken \& Jansz, 2006), are to be understood in their dialectical relation to various contextual factors. For instance, active mediation is likely to occur in families with young children across the age span of 3 to 9, but manifests itself as explanations and justifications towards the youngest ones rather than discussions. Other characteristics than age, like the child or parent's temperament or children's problematic media use, may equally influence the extent to which children are being heard. In addition, the child's exposure to media use of others (e.g., older siblings) is likely to determine the need for different or preventive (content) restriction measures, and this typically at an earlier age. Finally, as the wholeness approach in cultural-historical research does not only account for localized complexities and provides a perspective of the local-global that is dialectically framed instead, we argue that future investigations are necessary to understand how global factors like societal conditions play a part and shape localized PM practices.

To conclude, we acknowledge that we presented an exploratory study and could not fully exclude the possibility of social desirability effects, even though data triangulation had to minimize such biases. Nonetheless, we argue that the power of this study is in providing good examples of emergent PM practices inasmuch as it is generating hypotheses and new directions for further research.

\section{ORCID}

Bieke Zaman (D) http://orcid.org/0000-0002-1933-1438 


\section{Acknowledgements}

We would like to thank the parents and children who participated in this project, as well as the students who transcribed the interviews.

\section{Funding}

This MiX-ICON project has received the support of iMinds (Interdisciplinary Institute for Technology), a research institute founded by the Flemish Government. Companies and organizations involved in the project are Studio 100, Monkube, Larian, CUO, MICT, MM Lab, EDM, with project support of IWT.

\section{References}

Chaudron, S. (2015). Young children (0-8) and digital technology. A qualitative exploratory study across seven countries (EUR-Scientific and Technical Research Reports). Publications Office of the EU. Retrieved from http://publications.jrc.ec.europa.eu/repository/handle/ JRC93239

Clark, L. S. (2011a). A multi-grounded theory of parental mediation: Exploring the complementarity of qualitative and quantitative family communication research. In K. B. Jensen (Ed.), A handbook of media and communication research: Qualitative and quantitative methodologies (2nd edition). New York, NY: Routledge.

Clark, L. S. (2011b). Parental mediation theory for the digital age. Communication Theory, 21, 323-343.

Connell, S. L., Lauricella, A. R., \& Wartella, E. (2015). Parental co-use of media technology with their young children in the USA. Journal of Children and Media, 9, 5-21.

Correa, T. (2014). Bottom-up technology transmission within families: Exploring how youths influence their parents' digital media use with dyadic data. Journal of Communication, 64, 103-124.

Eastin, M. S., Greenberg, B. S., \& Hofschire, L. (2006). Parenting the Internet. Journal of Communication, 56, 486-504.

Fleer, M., Hedegaard, M., \& Tudge, J. (2012). World yearbook of education 2009: Childhood studies and the impact of globalization: Policies and practices at global and local levels. New York, NY: Routledge.

Gentile, D. A., Nathanson, A. I., Rasmussen, E. E., Reimer, R. A., \& Walsh, D. A. (2012). Do you see what I see? Parent and child reports of parental monitoring of media. Family Relations, 61, 470-487.

Hargittai, E., \& Hsieh, Y. P. (2012). Succinct survey measures of Web-use skills. Social Science Computer Review, 30, 95-107.

Holloway, D., Green, L., \& Livingstone, S. (2013). Zero to eight. Young Children and their Internet use. London, UK: LSE.

Jenkins, H. (2004). The cultural logic of media convergence. International Journal of Cultural Studies, 7, 33-43.

Lee, S.-J. (2013). Parental restrictive mediation of children's Internet use: Effective for what and for whom? New Media \& Society, 15, 466-481.

Livingstone, S., \& Helsper, E. J. (2008). Parental mediation of children's Internet use. Journal of Broadcasting \& Electronic Media, 52, 581-599.

Mendoza, K. (2009). Surveying parental mediation: Connections, challenges and questions for media literacy. Journal of Media Literacy Education, 1, $28-41$. 
Nathanson, A. I. (2015). Media and the family: Reflections and future directions. Journal of Children and Media, 9, 133-139.

Nikken, P., \& Jansz, J. (2006). Parental mediation of children's videogame playing: A comparison of the reports by parents and children. Learning, Media and Technology, 31, 181-202.

Nikken, P., \& Jansz, J. (2014). Developing scales to measure parental mediation of young children's Internet use. Learning, Media and Technology, 39, 250-266.

Nikken, P., Jansz, J., \& Schouwstra, S. (2007). Parents' interest in videogame ratings and content descriptors in relation to game mediation. European Journal of Communication, 22, 315336.

Ólafsson, K., Livingstone, S., \& Haddon, L. (2014). Children's use of online technologies in Europe. A review of the European evidence base. London, UK: LSE.

Padilla-Walker, L. M., Coyne, S. M., Fraser, A. M., Dyer, W. J., \& Yorgason, J. B. (2012). Parents and adolescents growing up in the digital age: Latent growth curve analysis of proactive media monitoring. Journal of Adolescence, 35, 1153-1165.

Plowman, L. (2014). Researching young children's everyday uses of technology in the family home. Interacting with Computers, 27, 36-46.

Plowman, L., \& McPake, J. (2013). Seven myths about young children and technology. Childhood Education, 89, 27-33.

Rodino-Colocino, M. (2007). Review article: Domesticity and new media. New Media \& Society, 9, 364-371.

Shin, W., \& Huh, J. (2011). Parental mediation of teenagers' video game playing: Antecedents and consequences. New Media \& Society, 13, 945-962.

Silverstone, R., \& Hirsch, E. (1992). Consuming technologies: Media and information in domestic spaces. London, UK: Routledge.

Sonck, N., Nikken, P., \& de Haan, J. (2012). Determinants of Internet mediation: A comparison of the reports by Dutch parents and children. Journal of Children and Media, 7, 96-113.

Valkenburg, P. M., Krcmar, M., Peeters, A. L., \& Marseille, N. M. (1999). Developing a scale to assess three styles of television mediation: "Instructive mediation," "restrictive mediation" and "social coviewing." Journal of Broadcasting \& Electronic Media, 43, 52-66.

Warren, R. (2001). In words and deeds: Parental involvement and mediation of children's television viewing. Journal of Family Communication, 1, 211-231. 


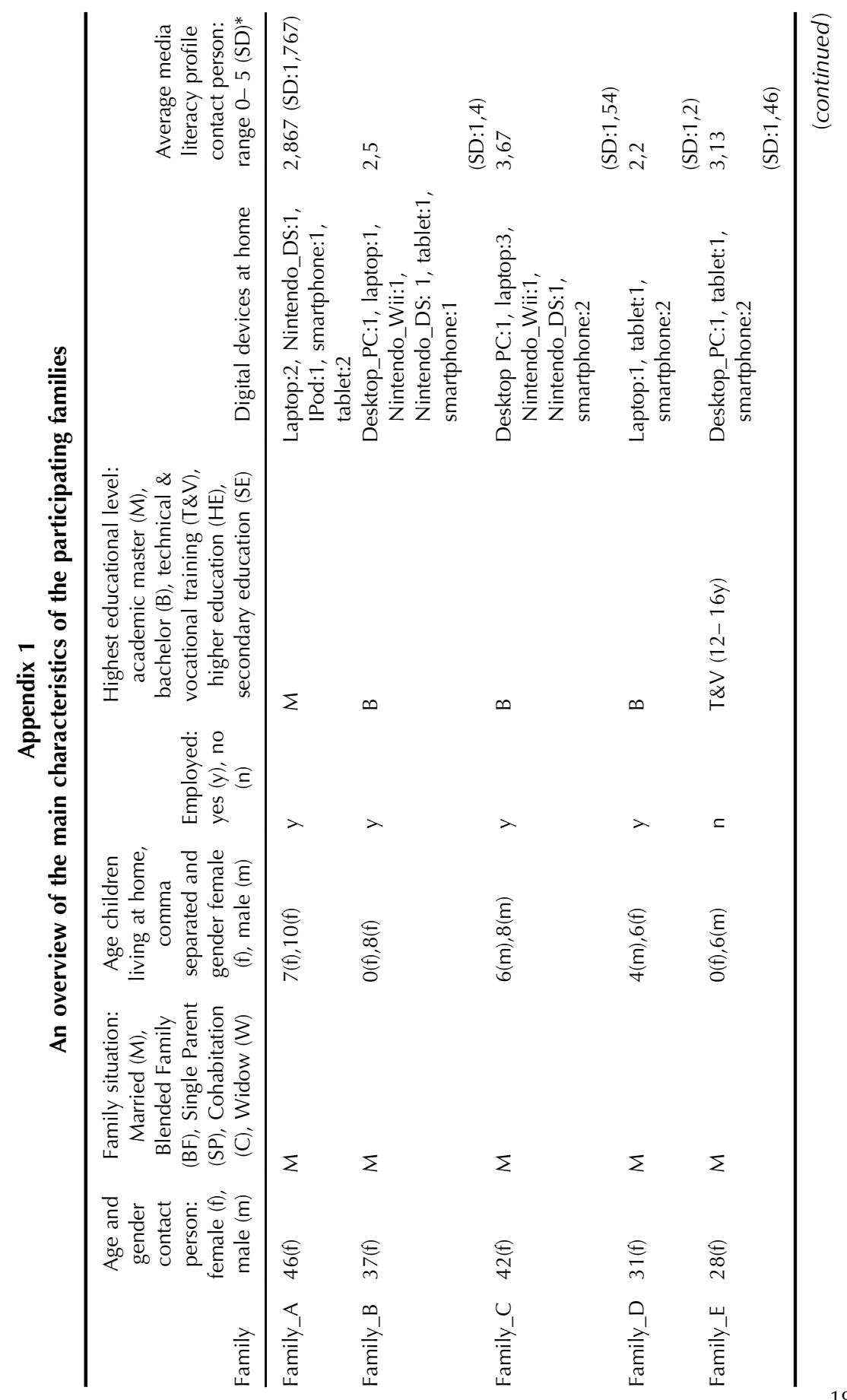




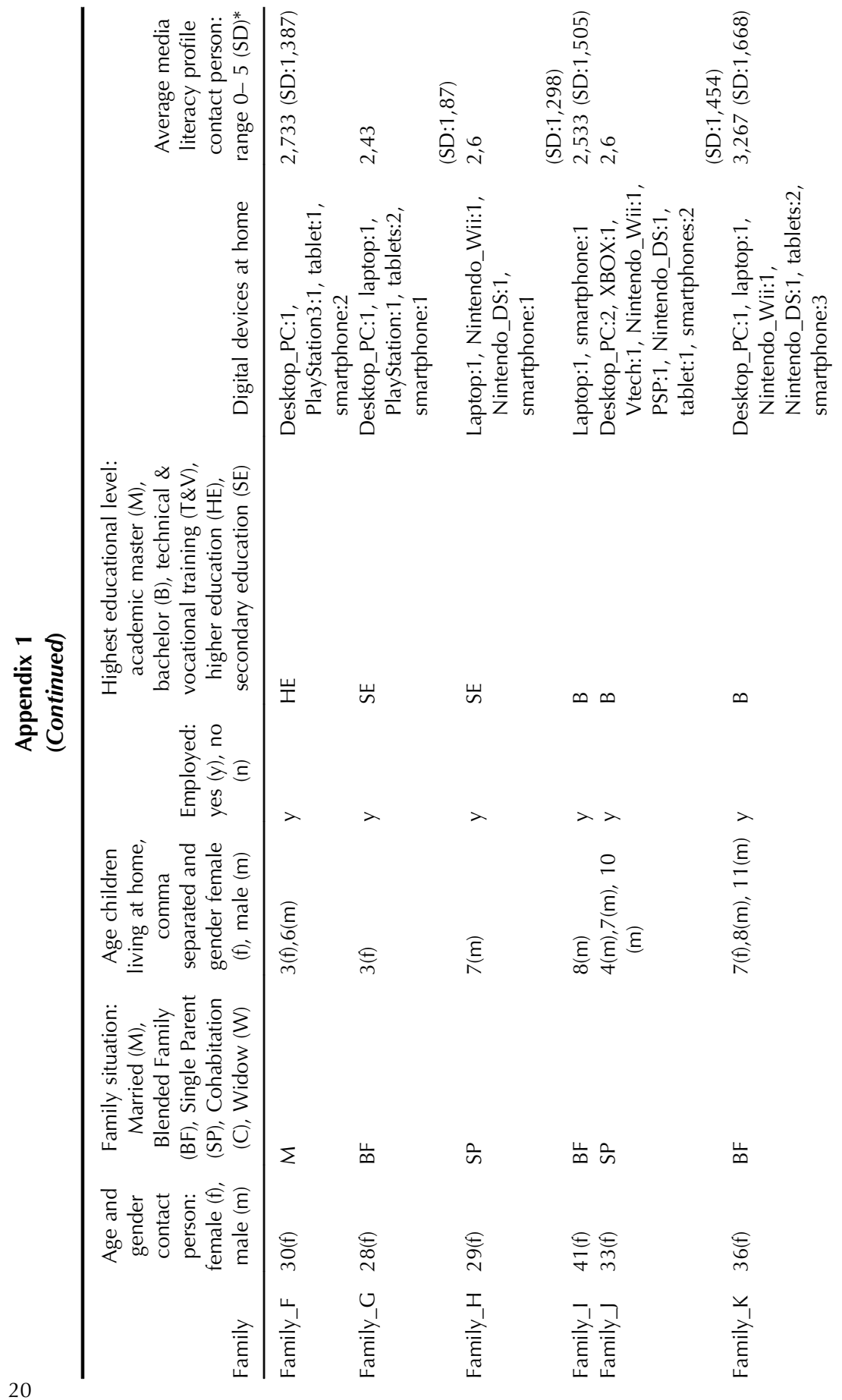



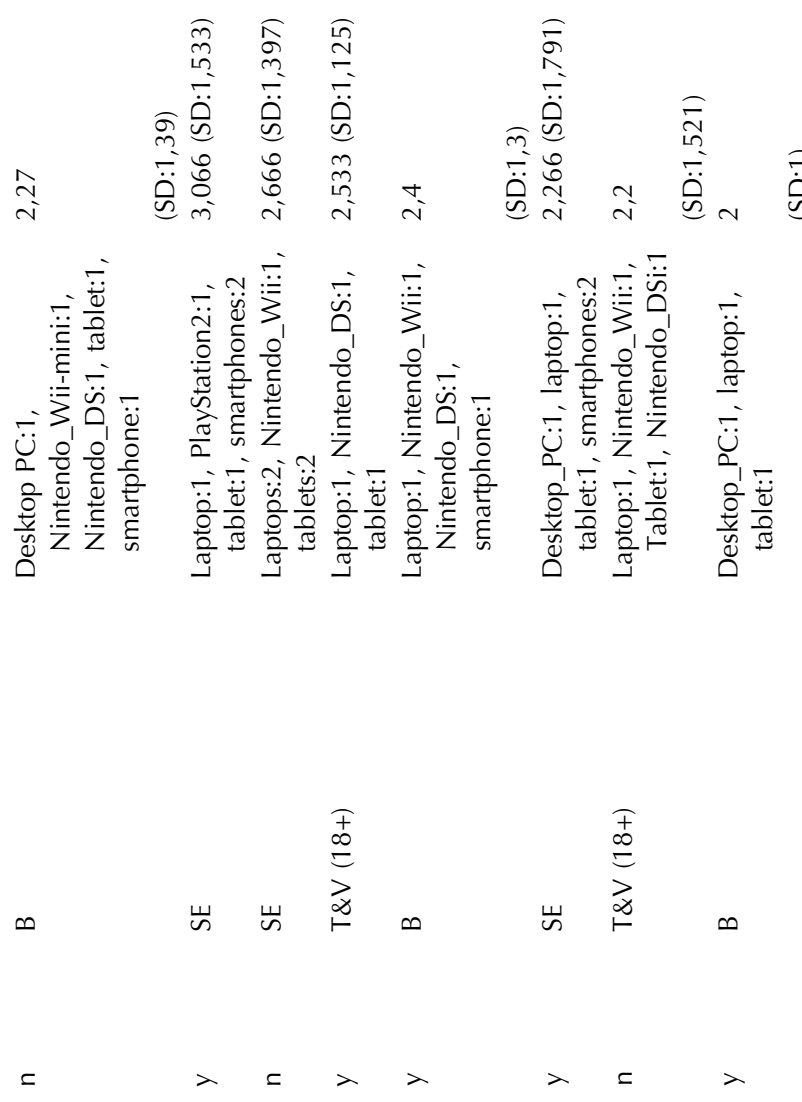

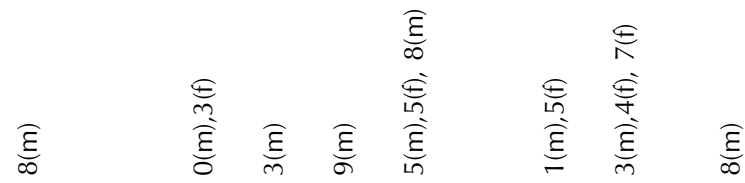

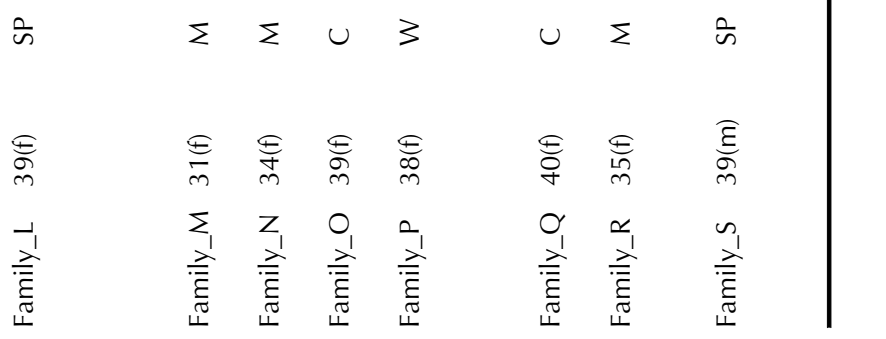




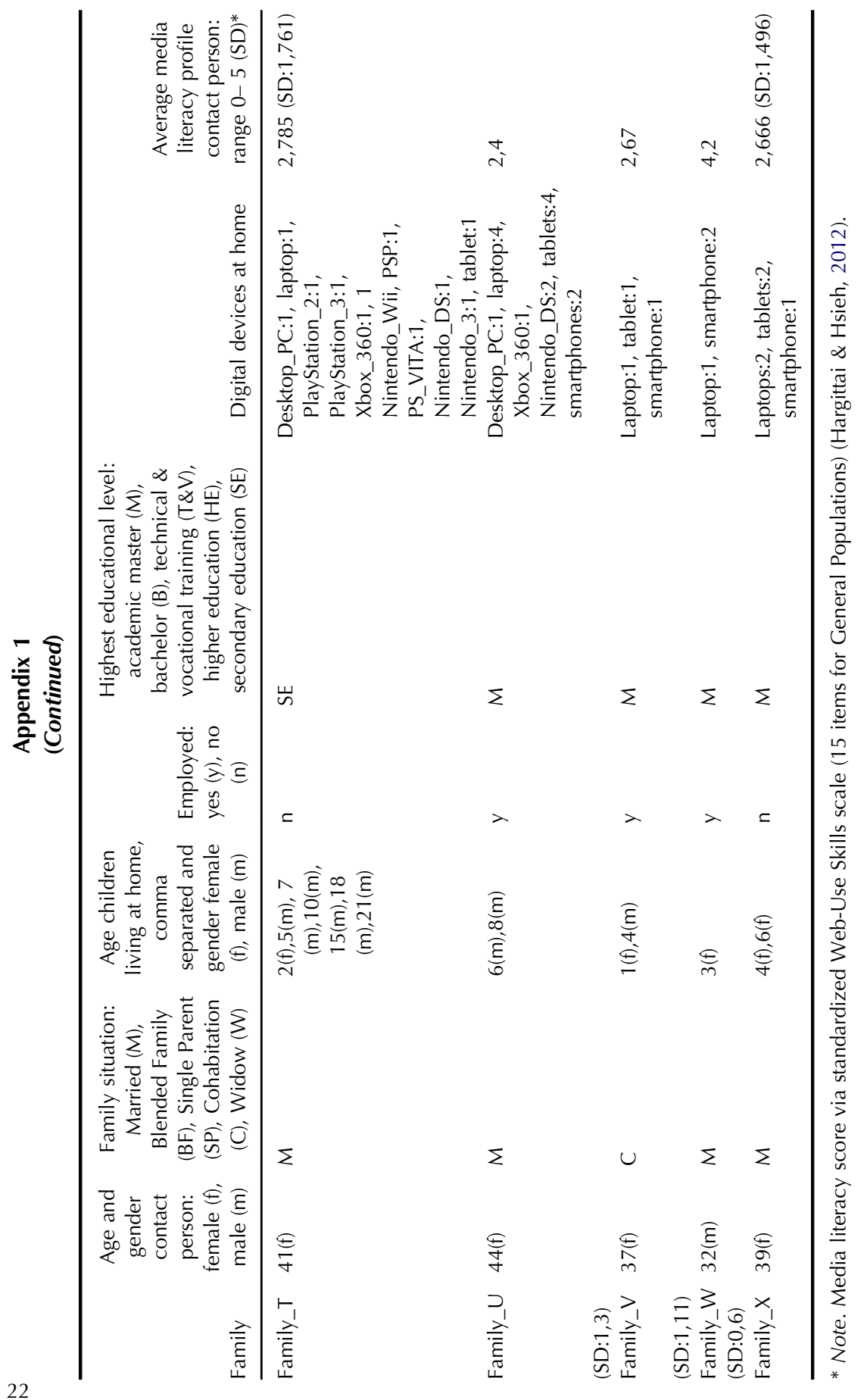

